\title{
Utility of Endoscopic Ultrasound with Bronchoscope-guided Fine-needle Aspiration for Detecting Driver Oncogenes in Non-small-cell Lung Cancer during Emergency Situations: Case Series
}

\author{
Koki Nakashima ${ }^{1,2}$, Yoshiki Demura ${ }^{1}$, Masahiro $\mathrm{Oi}^{1}$, Mio Tabata ${ }^{1}$, Toshihiko Tada ${ }^{1}$, \\ Kohei Shiozaki ${ }^{1}$, Masaya Akai ${ }^{1}$ and Tamotsu Ishizuka ${ }^{2}$
}

\begin{abstract}
:
Since it is difficult to obtain tumor tissue via airway observation for lung cancer patients with a poor respiratory condition, endoscopic ultrasound with bronchoscope-guided fine-needle-aspiration (EUS-B-FNA), a transesophageal procedure, is effective for such patients. We herein report three patients with driver oncogenes taken to the emergency department because of lung cancer-related symptoms. EUS-B-FNA was performed because of the patients' poor respiratory conditions to detect driver oncogenes. The general conditions improved, and the patients achieved a long-term survival with tyrosine kinase inhibitors. Our findings suggest that EUS-B-FNA should be considered to detect driver oncogenes in lung cancer patients despite poor respiratory conditions in emergency departments.
\end{abstract}

Key words: non-small-cell lung cancer, EUS-B-FNA, EGFR, ALK, driver oncogene, oncologic emergency

(Intern Med 60: 1061-1065, 2021)

(DOI: 10.2169/internalmedicine.5594-20)

\section{Introduction}

Lung cancer is the leading cause of cancer-related death worldwide (1). Non-small-cell lung cancer (NSCLC) accounts for approximately $80 \%$ of all lung cancers. Most cases are encountered in the unresectable and metastatic stage at the initial diagnosis (2). Furthermore, previous studies $(3,4)$ have reported that $13-40 \%$ of patients with lung cancer were diagnosed following emergency admission (DFEA). Fujimoto et al. (3) also reported that the performance status (PS) in the emergency department was not an independent predictor of the overall survival, while improvement of PS and epidermal growth factor receptor (EGFR) mutation positivity were independent predictors of overall survival. Thus, detecting driver oncogenes is beneficial for patients with NSCLC DFEA to achieve a long-term survival. However, due to their general or respiratory conditions, it is often difficult to obtain tumor tissues with airway observa- tion in such patients.

Several studies (5-8) have reported the utility and safety of endoscopic ultrasound with bronchoscope-guided fineneedle aspiration (EUS-B-FNA) in patients with lung cancer and poor general or respiratory conditions. These findings suggest that EUS-B-FNA might be an appropriate modality for managing patients with NSCLC DFEA.

To our knowledge, this is the first report on the utility of EUS-B-FNA for detecting driver oncogenes in patients with NSCLC DFEA during emergency situations.

\section{Case Reports}

\section{Procedures}

Each patient provided their written informed consent to undergo EUS-B-FNA. EUS-B-FNA was performed under local anesthesia with midazolam and fentanyl with the patients in the supine position. In EUS-B-FNA, a convex probe en-

${ }^{1}$ Department of Respiratory Medicine, Japanese Red Cross Fukui Hospital, Japan and ${ }^{2}$ Third Department of Internal Medicine, Faculty of Medical Sciences, University of Fukui, Japan

Received: June 12, 2020; Accepted: September 15, 2020; Advance Publication by J-STAGE: November 2, 2020

Correspondence to Dr. Koki Nakashima, kouk0527@yahoo.co.jp 
Table. Details of EUS-B-FNA.

\begin{tabular}{lcccccc}
\hline & PS & $\begin{array}{c}\text { Key reason for } \\
\text { EUS-B-FNA }\end{array}$ & $\begin{array}{c}\text { Days from first } \\
\text { visit (days) }\end{array}$ & $\begin{array}{c}\text { Punctured } \\
\text { lesions }\end{array}$ & $\begin{array}{c}\text { Total number of } \\
\text { passes (times) }\end{array}$ & $\begin{array}{c}\text { Procedure time (*) } \\
(\text { minutes) }\end{array}$ \\
\hline $\begin{array}{l}\text { Case } 1 \\
43 \text { yo, F }\end{array}$ & 3 & respiratory failure & 2 & \#4L+\#7 & 9 & 20 \\
$\begin{array}{l}\text { Case 2 } \\
75 \text { yo, F }\end{array}$ & 3 & respiratory failure & 0 & no & no \\
$\begin{array}{l}\text { Case 3 } \\
28 \text { yo, M }\end{array}$ & 3 & $\begin{array}{c}\text { poor respiratory } \\
\text { condition (cough) }\end{array}$ & 2 & \#4L & 7 & 22 \\
\hline
\end{tabular}

* Procedure time is defined as the time from the first insertion of the bronchoscope to the end of the procedures.

EUS-B-FNA: endoscopic ultrasound with bronchoscope-guided fine-needle aspiration, PS: performance status, yo: years old, F: female, M: male

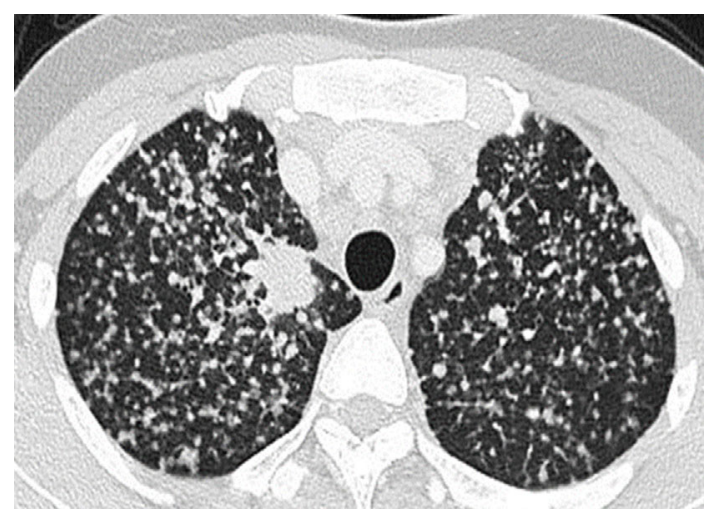

Figure 1. Chest computed tomography performed at the emergency department showing diffuse micronodules.

dobronchial ultrasonography (CP-EBUS) endoscope (BF-UC 260F-OL8; Olympus, Tokyo, Japan) was inserted and advanced through the esophagus. After confirming the structure and blood vessels using ultrasound with Doppler flow, the lesions were punctured with a 22-gauge EBUS needle (NA-201SX-4022 or NA-U401SX-4022; Olympus) under real-time ultrasonographic guidance. In all cases, both the EGFR mutation and anaplastic lymphoma kinase (ALK) gene were evaluated. Polymerase chain reaction (PCR) with fine-needle aspiration cytology was performed to evaluate the EGFR mutation, and immunohistochemistry (IHC) screening was used for $A L K$ gene tests. If the IHC results were positive, further verification with fluorescence in situ hybridization (FISH) was done. To prevent complications, EUS-B-FNA was performed under conditions of 'nothing by mouth' for at least five hours, and the patients were permitted oral intake on the day after the procedure. Furthermore, all patients were administered antibiotics with $2 \mathrm{~g}$ ceftriaxone sodium hydrate before and after the procedures as well as on the day after the procedure.

All procedures were performed by pulmonologists at our institution. A rapid on-site cytological evaluation (ROSE) was not performed. The details of the procedures are shown in Table.

\section{Case 1}

A 43-year-old woman who had never smoked presented to the emergency department with dyspnea and respiratory

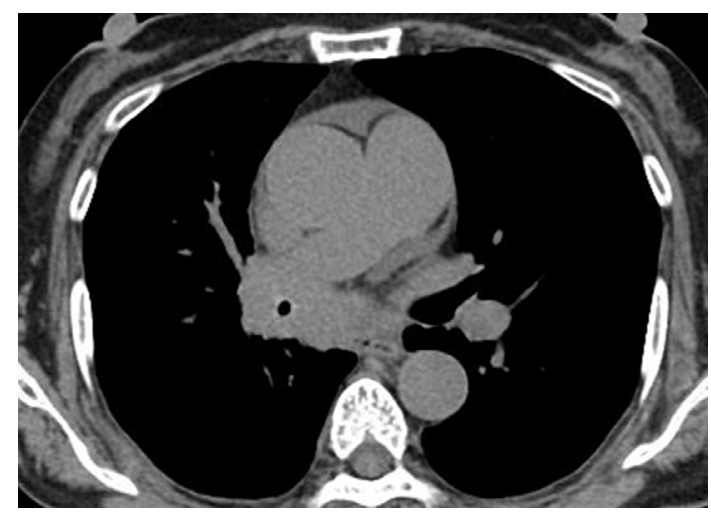

Figure 2. Chest computed tomography at the emergency department showing mediastinal lymph node swelling with stenosis of the right main bronchus.

failure. Her arterial oxygen partial pressure $\left(\mathrm{PaO}_{2}\right)$ was 52.8 Torr on room air. She could not walk due to dyspnea and back pain and had an Eastern Cooperative Oncology Group (ECOG) PS of 3. Imaging findings showed multiple brain, bone, and pulmonary metastases (Fig. 1). Two days after admission to the emergency department, EUS-B-FNA of the \#4L lymph node was performed, and a diagnosis of lung adenocarcinoma was made, with PCR showing an EGFR (exon 19 deletion) mutation. Furthermore, IHC showed that the $A L K$ gene was negative. Three days later, afatinib (20 $\mathrm{mg} /$ day) was administered as first-line treatment, and her PS improved from 3 to 1 , with improvement in her respiratory failure also seen. The best overall response was partial response. Afatinib treatment was continued for 18 months until disease progression, and the survival achieved was 28 months.

\section{Case 2}

A 75-year-old woman with uncontrollable asthma-chronic obstructive pulmonary disease overlap (ACO) was admitted to the emergency department because of dyspnea and respiratory failure, with a $\mathrm{PaO}_{2}$ of 53.6 Torr and arterial carbon dioxide partial pressure $\left(\mathrm{PaCO}_{2}\right)$ of 51.1 Torr on room air. The patient could not walk due to dyspnea and back pain and had an ECOG PS of 3. Imaging findings showed a primary tumor on the right lower lobe, right endobronchial stenosis caused by swelling of the mediastinal lymph nodes, 


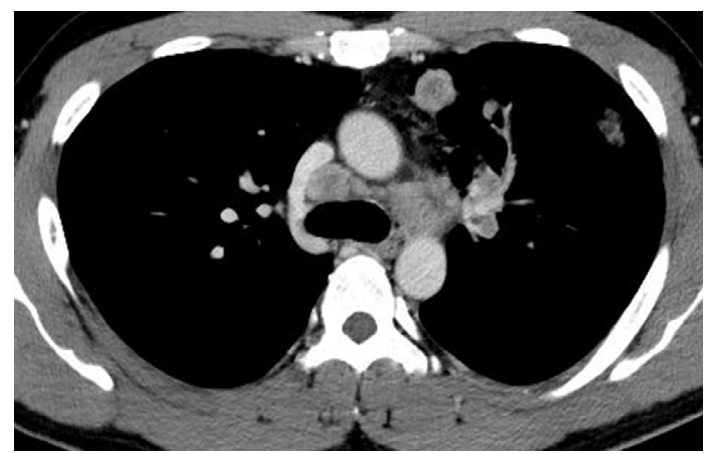

Figure 3. Chest computed tomography at the emergency department showing a primary tumor in the left upper lobe and swelling of the mediastinal and hilar lymph nodes.

adrenal metastasis, and multiple brain and bone metastases (Fig. 2). On the day of admission to the emergency department, EUS-B-FNA of the \#7 lymph node was performed, and a diagnosis of lung adenocarcinoma was made, with PCR showing an EGFR (exon 19 deletion) mutation. Furthermore, IHC showed that the $A L K$ gene was negative, and reverse transcription PCR also showed that the c-ros oncogene 1 receptor tyrosine kinase $(R O S-1)$ gene negative. While waiting for the examination results, pain was managed with analgesics and ACO was managed with inhaled medication. Four days later, osimertinib (80 mg/day) was administered as first-line treatment, and her PS improved from 3 to 1, with improvement in her respiratory failure also seen. At Present, 13 months have passed since her first visit to our hospital, and osimertinib treatment is ongoing without disease progression.

\section{Case 3}

A 28-year-old man who had never smoked presented to the emergency department with hemoptysis and severe cough and had an ECOG PS of 3. Imaging findings showed a primary tumor on the left upper lobe, swelling of the mediastinal and hilar lymph nodes, and liver and multiple bone metastases (Fig. 3). Two days after admission to the emergency department, EUS-B-FNA of the \#4L lymph node was performed, and a diagnosis of lung adenocarcinoma was made, with both IHC and FISH showing $A L K$ gene positivity. Alectinib (600 mg/day) was administered as first-line treatment, and his PS improved from 3 to 0 . The best overall response was complete response. The patient received alectinib treatment for 20 months until disease progression, and the survival achieved was 39 months.

\section{Discussion}

To our knowledge, this is the first report on the utility of EUS-B-FNA for detecting driver oncogenes in patients with NSCLC DFEA during emergency situations. The courses of the present cases suggest two important clinical issues.

First, EUS-B-FNA is an effective and safe approach that is capable of obtaining tumor tissue specimens from patients with NSCLC DFEA despite a poor general or respiratory condition. Several studies (5-9) have reported the efficacy and safety of EUS-(B-)FNA in critically ill patients, including for those on mechanical ventilation. Oki et al. (10) revealed that EUS-B-FNA has advantages of fewer doses of anesthetics and sedatives, a shorter procedure time, and fewer instances of oxygen desaturation during the procedure than endobronchial ultrasound-guided transbronchial needle aspiration (EBUS-TBNA). EUS-B-FNA is thus considered an appropriate approach for such patients. Furthermore, Araya et al. (9) reported that EUS-B-FNA is a practical and feasible method for obtaining tumor tissue to detect EGFR mutations and $A L K$ fusion genes. In the present case series, we selected EUS-B-FNA because airway observation, such as EBUS-TBNA or transbronchial biopsy (TBB), was difficult in these patients due to poor respiratory condition. As a result, we were able to rapidly and safely diagnose driver oncogene-positive lung adenocarcinoma with EUS-B-FNA. Furthermore, no complications were observed. We therefore believe that EUS-B-FNA is an effective and safe procedure for detecting driver oncogenes in patients with NSCLC DFEA during emergency situations.

Second, treatments with tyrosine kinase inhibitors (TKIs) are beneficial for patients with NSCLC DFEA despite a poor general or respiratory condition. Fujimoto et al. (3) reported that improvement in the PS and EGFR mutation positivity were independent predictors of the overall survival in patients with NSCLC DFEA. Inoue et al. (11) reported that EGFR mutation-positive patients with an extremely poor PS benefit from first-line gefitinib treatment, so checking for EGFR mutations was strongly recommended for these patients. Iwama et al. (12) revealed that alectinib was effective in patients with NSCLC with $A L K$ rearrangement positivity and poor PS, for whom chemotherapy was not indicated. Several studies (13-17) also reported the efficacy and safety of TKIs for such patients. Based on these results, the guideline for lung cancer in Japan recommends that treatments with TKIs should be considered for patients with poor PS driver oncogenes (18). In the present case series, rapid therapeutic intervention with TKIs led to an improvement in the general conditions and a long-term survival. This finding indicates that treatments with TKIs should be considered even when patients with driver oncogenes have a poor general condition on admission.

Given the above, our present findings suggest that EUSB-FNA is an effective and safe procedure for detecting driver oncogenes in patients with NSCLC DFEA, regardless of poor respiratory conditions. Furthermore, appropriate treatment with TKIs should be performed to improve the PS dramatically and achieve a long-term survival despite patients with driver oncogenes having a poor general condition on admission.

Recently, next-generation sequencing (NGS), a newly developed platform, has enabled the simultaneous detection of numerous genetic alterations of tens to hundreds of genes $(19,20)$. Although several studies (21-23) have shown 
that EBUS-TBNA samples are sufficient for NGS analyses, only few studies on EUS-B-FNA have been performed. However, the needles used with EBUS-TBNA and EUS-BFNA are the same; therefore, EUS-B-FNA samples may also be sufficient for NGS analyses. Further studies are needed to evaluate the validity of EUS-B-FNA specimens for NGS analyses.

EUS-B-FNA is an effective modality recommended by some guidelines $(18,24,25)$. However, it is difficult to say that it is widely used, since it is inaccessible for pulmonologists to perform the transesophageal method. In general, EUS-B-FNA is a safe method, with a meta-analysis (26) showing that there were no severe complications associated. Furthermore, using this method leads to wider specimen collection area, even if the target lesions are inaccessible by other transbronchial modalities, such as \#8 lymph nodes. We believe, therefore, that EUS-B-FNA should be used more widely.

\section{Conclusion}

EUS-B-FNA is an effective and safe procedure for detecting driver oncogenes in patients with NSCLC DFEA despite a poor respiratory condition. Appropriate treatments with TKIs should be performed to improve the PS and achieve a long-term survival even when patients with driver oncogenes have a poor general condition at admission.

The authors state that they have no Conflict of Interest (COI).

\section{References}

1. Sigel RL, Miller KD, Jemal A. Cancer statistics, 2015. CA Cancer J Clin 65: 5-29, 2015.

2. Molina JR, Yang P, Cassivi SD, et al. Non-small cell lung cancer: epidemiology, risk factors, treatment, and survivorship. Mayo Clin Proc 83: 584-594, 2008.

3. Fujimoto D, Shimizu R, Morimoto T, et al. Analysis of advanced lung cancer patients diagnosed following emergency admission. Eur Respir J 45: 1098-1107, 2015.

4. Elliss-Brookes L, McPhail S, Ives A, et al. Routes to diagnosis for cancer - determining the patient journey using multiple routine data sets. Br J Cancer 107: 1220-1226, 2012.

5. Nakashima K, Demura Y, Oi M, et al. Usefulness and safety of endoscopic ultrasound with bronchoscope-guided fine needle aspiration for the diagnosis of lung cancer. J Jpn Soc Respir Endosc 42: 129-133, 2020 (in Japanese, Abstract in English).

6. Prasad KT, Sehgal IS, Gupta N, et al. Endoscopic ultrasound (with an echobronchoscope)-guided fine-needle aspiration for diagnosis if a mediastinal lesion in a mechanically ventilated patient: a case report and systematic review of the literature. Indian J Crit Care Med 20: 608-612, 2016.

7. Abouzgheib W, Shweihat Y, Bartter T. Oesophageal applications of the convex curvilinear ultrasound bronchoscope; an illustrative case series. Respirology 16: 965-968, 2011.

8. Mohamadnejad M, Leblanc JK, Sherman S, et al. Bedside endoscopic ultrasound in critically Ill patients. Diagn Ther Endosc 2011: 529791, 2011.

9. Araya T, Demura Y, Kasahara K, et al. Usefulness of transesophageal bronchoscopic ultrasound-guided fine-needle aspiration in the pathologic and molecular diagnosis of lung cancer lesions adjacent to the esophagus. J Bronchology Interv Pulmonol 20: 121-126, 2013.

10. Oki M, Saka H, Ando M, et al. Transbronchial vs transesophageal needle aspiration using an ultrasound bronchoscope for the diagnosis of mediastinal lesions: a randomaized study. Chest 147: 1259-1266, 2015.

11. Inoue A, Kobayashi K, Usui K, et al. First-line gefitinib for patients with advanced non-small-cell lung cancer harboring epidermal growth factor receptor mutations without indication for chemotherapy. J Clin Oncol 27: 1394-1400, 2009.

12. Iwama $\mathrm{E}$, Goto $\mathrm{Y}$, Murakami $\mathrm{H}$, et al. Alectinib for patients with ALK rearrangement-positive non-small cell lung cancer and a poor performance status (Lung Oncology Group in Kyushu 1401). J Thorac Oncol 12: 1161-1166, 2017.

13. Zhou C, Wu YL, Chen G, et al. Erlotinib versus chemotherapy as first-line treatment for patients with advanced EGFR mutationpositive non-small-cell lung cancer (OPTIMAL, CTONG-0802): a multicentre, open-label, randomised, phase 3 study. Lancet Oncol 12: 735-742, 2011.

14. Rosell R, Carcereny E, Gervais R, et al. Erlotinib versus standard chemotherapy as first-line treatment for European patients with advanced EGFR mutation-positive non-small-cell lung cancer (EURTAC): a multicentre, open-label, randomised phase 3 trial. Lancet Oncol 13: 239-246, 2012.

15. Solomon BJ, Mok T, Kim DW, et al. First-line crizotinib versus chemotherapy in ALK-positive lung cancer. N Engl J Med 371: 2167-2177, 2014.

16. Wu YL, Lu S, Lu Y, et al. Results of PROFILE 1029, a phase III comparison of first-line crizotinib versus chemotherapy in East Asian patients with ALK-positive advanced non-small cell lung cancer. J Thorac Oncol 13: 1539-1548, 2018.

17. Araya T, Demura Y, Kasahara K, et al. Successful treatments with a combination of electrocautery using wire snares and gefitinib in patients with EGFR-mutant lung cancer and central airway obstruction. Intern Med 52: 2331-2335, 2013.

18. The Japan Lung Cancer Society. Guidelines for Diagnosis and Treatment of the Lung Cancer 2018. Kanehara, ToKyo, 2018: 4143, 161-162, 167.

19. Goodwin S, McPherson JD, McCombie WR. Coming of age: ten years of next-generation sequencing technologies. Nat Rev Genet 17: 333-351, 2016.

20. Serratì S, De Summa S, Pilato B, et al. Next-generation sequencing: advances and applications in cancer diagnosis. Onco Targets Ther 9: 7355-7365, 2016.

21. Fangfang X, Xiaoxuan Z, Xiaowei M, et al. Next-generation sequencing for genotyping of endobronchial ultrasound-guided transbronchial needle aspiration samples in lung cancer. Ann Thorac Surg 108: 219-226, 2019.

22. Scott M, Janakiraman S, Esma G, et al. Performance of nextgeneration sequencing on small tumor specimens and/or low tumor content samples using a commercially available platform. PLoS One 13: 0196556, 2018.

23. Kanagal-Shamanna R, Portier BP, Singh RR, et al. Nextgeneration sequencing-based multi-gene mutation profiling of solid tumors using fine needle aspiration samples: promises and challenges for routine clinical diagnostics. Mod Pathol 27: 314-327, 2014.

24. Silvestri GA, Gonzalez AV, Jantz MA, et al. Methods for staging non-small cell lung cancer: diagnosis and management of lung cancer, 3rd ed: American College of Chest Physicians evidencebased clinical practice guidelines. Chest 143: e211S-e250S, 2013.

25. Vilmann P, Clementsen PF, Colella $S$, et al. Combined endobronchial and esophageal endosonography for the diagnosis and staging of lung cancer: European Society of Gastrointestinal Endoscopy (ESGE) Guideline, in cooperation with the European Respiratory Society (ERS) and the European Society of Thoracic Sur- 
geons (ESTS). Endoscopy 47: 545-559, 2015.

26. Dhooria S, Aggarwal AN, Gupta D, Behera D, Agarwal R. Utility and safety of endoscopic ultrasound with bronchoscope-guided fine-needle aspiration in mediastinal lymph node sampling: systematic review and meta-analysis. Respir Care 60: 1040-1050, 2015 .
The Internal Medicine is an Open Access journal distributed under the Creative Commons Attribution-NonCommercial-NoDerivatives 4.0 International License. To view the details of this license, please visit (https://creativecommons.org/licenses/ by-nc-nd/4.0/).

(C) 2021 The Japanese Society of Internal Medicine Intern Med 60: 1061-1065, 2021 